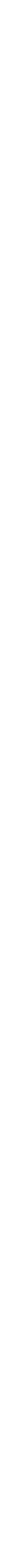




\title{
Energetic and structural properties of different conformations of merocyanine and its protonated forms
}

\author{
Rahul Singh ${ }^{1}$, Michael C. Böhm ${ }^{2}$ and Ganesh Balasubramanian ${ }^{1, \Psi}$ \\ ${ }^{1}$ Department of Mechanical Engineering, Iowa State University, Ames, IA 50011, USA \\ ${ }^{2}$ Eduard-Zintl-Institut für Anorganische und Physikalische Chemie, Technische Universität \\ Darmstadt, D-64287 Darmstadt, Germany
}

\footnotetext{
${ }^{\Psi}$ Corresponding author

Address: 2092 Black Engineering Building, Ames, IA 50011, USA.

Phone: 515-294-9226, Email: bganesh@iastate.edu 


\section{Abstract}

We investigate the conformational stabilities, dipole moments, bond lengths and partial charges of photo-sensitive spiropyran (1',3'-dihydro-1',3',3'-trimethyl-6-nitro-spiro[2H-1-benzopyran$2,2^{\prime}-[2 \mathrm{H}]$ indole]) after its photo-switching to the open form conformers, merocyanine and its protonated counterparts. Two approaches, an ab initio Hartree-Fock method supplemented by the Møller-Plesset second-order perturbation theory and density functional theory under B3LYP parameterization, are employed with extended basis sets to examine the energetic ordering of the conformers in vacuum and in an aqueous environment. The influence of the solvent on the conformers is demonstrated by the notable changes in the structural and electrostatic properties.

Keywords: Photoresponsive merocyanine, protonation, ab initio MP2, DFT, solvent effects, partial charges, dipole moment 


\section{Introduction}

Spiropyran (SP) systems are well-known chromophores that reversibly photoisomerize to the conjugated merocyanine (MC) moieties,[1-4] which are attractive candidates for designing devices for optical switching and storage, sensors, cell imaging, etc.[5-9] SP absorbs light in the wavelength range of 200-400 nm to induce bond-cleavage producing the open form MC (Fig. 1). The reverse transformation occurs by either thermal relaxation or light stimulus in the visible range.[10] These conversions from SP to planar conjugate MC forms as well as the reverse processes are accompanied by changes in the optical, polarization, and transport properties. Literature on this isomerization pathway[11-14] suggests that the ring opening of SP proceeds through several steps involving MC conformers as intermediates. Excluding the transition states, six nearly planar conformers of the open structure can be formed by rotation around the three central bonds $\mathrm{C} 3-\mathrm{C} 4, \mathrm{C} 4-\mathrm{C} 5$ and $\mathrm{C} 5-\mathrm{C} 6$. The pathway is influenced by the proximity of the $\mathrm{O}$ atom of the benzopyran part with the methyl groups of the dihydroindole moiety. The six configurations of MC differ in the combination of cis (C) and trans (T) arrangements mapped by the angular orientations $\left(\alpha_{1}, \alpha_{2}\right.$ and $\left.\alpha_{3}\right)$ as shown in Fig. 1. In MC and its protonated forms the configurations are denoted as CCC, TCC, CTC, CTT, TTT, and TTC according to relative angular orientations. SP-MC derivatives have been synthesized in the past by performing stimulus sensitive conversions to the protonated $\mathrm{MCH}^{+}$form via a variation of the $\mathrm{pH}$ value.[1517] An earlier investigation on the thermal isomerization of nitro-spiropyran[18] shows that protonation of SP on the $\mathrm{N}$ atom stabilizes the closed form and makes it the more dominant product in the photochromic reaction. Protonation on the $\mathrm{O}$ site (i.e. $\mathrm{O} 8$ in the six-membered ring; see Fig. 1), however, leads to an opening of the bond (C3 - O8) and SP, becoming unstable, converts into merocyanine.

Large-scale numerical simulations of complex molecules, such as polymers containing SP-MC chromophores as side chains, are limited by the accuracy of the employed force field parameters. $A b$ initio quantum chemical calculations, though computationally expensive, provide detailed descriptions of the electronic structure of these photoresponsive moieties that can serve as a tool to improve force fields for molecular computations. While the photo-stimulated isomerization of various SP derivatives in different media (gas phase, solution, mono-layers, polymers) has been investigated extensively both by experiments[19-23] and computations,[13,24,25] literature on 
the effects of protonation and on structural properties of $\mathrm{MCH}^{+}$are sparse. In the present work we employ ab initio and density functional (DFT) calculations on $\mathrm{MC}$ and $\mathrm{MCH}^{+}$conformers to understand the role of protonation on the molecular geometry and the electronic structure of the chromophores in the gas phase (i.e. in vacuum) as well as in an aqueous environment.

\section{Computational Methods}

Quantum chemical post-Hartree-Fock (HF) Møller-Plesset (MP2) theory is employed to optimize the ground state structures of the open form MCs and the protonated forms. They are derived from 1',3'-dihydro-1',3',3'-trimethyl-6-nitro-spiro[2H-1-benzopyran-2,2'-[2H]indole].[26,27] The post HF results are compared with findings from DFT-B3LYP calculations of MC, already presented in our previous report,[25] and its protonated conformers. Recent computational investigations $[17,25]$ on this photoisomerization reaction discussed the energetic ordering of the MC conformers under different points of view. Most of these studies evaluated the molecular and electronic properties using the density functional approaches. To compare DFT results with its semi-empirical parameters against ab initio findings we have performed HF calculations supplemented by the MP2 correlation scheme. In our recent publication[25] the influence of the chosen exchange-correlation potential in DFT calculations has been investigated. This comparative study has shown that the B3LYP method provides the most reliable results. As a matter of fact we have restricted the present investigation to this potential. In this letter we demonstrate that the two methods (MP2, DFT) result in different energy landscapes. The present results emphasize that even with the help of elaborate quantum chemical approaches the optimization of force fields for systems with quasi-degenerate configurations is a challenging task. Our calculations are repeated with the molecules in an aqueous environment modeled by the polarizable continuum model (PCM).[28] We have used the PCM method in combination with the 6-31G(d) basis set. $\mathrm{A} \mathrm{Cl}^{-}$ion is included for the $\mathrm{MCH}^{+}$systems in vacuum as well as in water to ensure charge neutrality; we use the label $\mathrm{MCH}^{+} \mathrm{Cl}^{-}$to denote this neutral cation - anion pair. Upon geometry optimization, we find that the $\mathrm{Cl}^{-}$resides at a distance of $\sim 250-350 \mathrm{pm}$ from the $\mathrm{MCH}^{+}$conformer in vacuum, while for the simulations in water the chloride ion is farther apart and is located at $\sim 1500-2000 \mathrm{pm}$ from the cation. In vacuum, $\mathrm{Cl}^{-}$is closest to $\mathrm{N} 2$ (shown in Fig.1) and the carbon atoms of the benzene ring bonded to N2. 

compared against those performed with a larger $6-311 \mathrm{G}(\mathrm{d}, \mathrm{p})++$ basis set. The latter basis contains d type and $\mathrm{p}$ type polarization functions to reduce the effect of the non-uniform electric field arising from the distorted environment of electrons. The additional diffuse functions offer an improved description of the electron distribution around the nucleus. But as we show later, the inclusion of polarization and diffuse functions does not lead to sizeable differences in the energetic ordering in comparison to calculations with the smaller basis set. Thus, it seems that the size of basis sets is of only minor importance in the prediction of relative energies of the considered conformers. Single-point calculations are performed to calculate electronic energies, dipole moments, bond lengths and partial charges on the optimized geometry of the most stable structures. The partial atomic charges are calculated with the Singh-Kollman method.[29]

\section{Results and discussion}

The energetic ordering in the electronic ground state of the different $\mathrm{MC}, \mathrm{MCH}^{+} \mathrm{Cl}^{-}$and $\mathrm{MCH}^{+}$ conformers is given in Tables 1 and 2. The MP2 and DFT energies of the optimized geometries of the six conformers for these three species in vacuum and for the two neutral systems in water are presented relative to the ground state energy of the TTC structure, which is the most stable neutral MC conformer (6-31G(d) basis set). The DFT results for the MC conformers are referenced from our earlier publication.[25] Given the nearly planar configurations of merocyanine molecules as mentioned above, it might be expected a priori that the CCC form is unstable and might isomerize to a structure with preferential trans arrangements. While this is observed in the DFT framework irrespective of the medium and the basis set adopted, the MP2 formalism predicts a strong stabilization of the CCC form relative to the other conformers. This effect is found both in the $\mathrm{MC}$ form and $\mathrm{MCH}^{+} \mathrm{Cl}^{-}$molecules. The TTC conformer is the most stable MC form in almost all computational approaches, while TTT has the lowest ground state energy in the majority of DFT calculations of the protonated $\mathrm{MCH}^{+} \mathrm{Cl}^{-}$conformers. Our findings, in concurrence with earlier reports,[17,18] predict that the TTC and TTT conformers are the most stable protonated $\mathrm{MCH}^{+}$structures (see Table 2) while TCC is energetically the least favorable. For the CTT isomer of MC the situation is rather complex. Within the DFT framework it is close in energy to the TTC conformation. This is valid both in vacuum as well as in water. In the MP2 vacuum calculation it is destabilized relative to TTC and TTT. Let us now 
come to the characteristic differences between the neutral $\mathrm{MCH}^{+} \mathrm{Cl}^{-}$and $\mathrm{MCH}^{+}$results presented in Table 2.. In vacuum, the stability sequence is observed to be different for the protonated case than the neutral form. We find either one (in MP2) or two (in DFT) isomers that are more stable than TTC with TTT being the most stable one in the majority of calculations.

Next, we compare results of MP2 calculations with predictions from DFT computations in larger detail. Tables 1 and 2 provide the energetic ordering as a function of the adopted approach and the chosen basis set. Both methods show that the TTT arrangement is energetically one of the most favorable conformers, especially in the protonated form. A major difference between the two methods arises in the prediction of other stable structures for $\mathrm{MC}, \mathrm{MCH}^{+} \mathrm{Cl}^{-}$and $\mathrm{MCH}^{+}$in vacuum. According to density functional theory CCC has unfavorable ground state energy for MC. In the MP2 vacuum calculation equipped with the larger basis set the CCC form of MC is significantly lower in energy than all other conformers. DFT and MP2 thus lead to completely contradictory estimates for the relative $\mathrm{MC}$ energies. Likewise for $\mathrm{MCH}^{+} \mathrm{Cl}^{-}$, as shown in Table 2, we see similar differences in the energies for the CCC isomer that is obtained from both the methods. On the other hand, both methods predict the $\mathrm{CCC}$ form of $\mathrm{MCH}^{+}$in vacuum to be energetically unfavorable. By comparison, one can see the contribution of an ionic pair in enabling a molecule towards an energetically favorable state. However, both DFT and MP2 calculations in vacuum identify $\mathrm{CTC}$ form of $\mathrm{MCH}^{+} \mathrm{Cl}^{-}$as a stable structure. While some ambiguity exists in asserting the relative energies of the conformational forms, improved correlation schemes might be necessary to provide results of higher accuracy, which is beyond the scope of this letter.

We have reported the stability ordering in three merocyanine systems in two levels of theory, DFT and MP2. The quantum chemical results unfortunately have shown that a physically reliable interpretation of the differences in the DFT and MP2 relative energies is still missing and that the present letter should be accepted only as a first attempt to compare unprotonated and protonated merocyanines. The ground state energies of the $\mathrm{MC}$ and $\mathrm{MCH}^{+} \mathrm{Cl}^{-}$conformers are also computed under an aqueous environment. All the $\mathrm{MC}$ and $\mathrm{MCH}^{+} \mathrm{Cl}^{-}$conformers become energetically more favorable than in the gas phase as reflected from the stabilization energies presented in Table 3. We also refer to our previous contribution[25] where we have derived the solvent dependent stabilization of the different MC systems. As we can see, the energetic ordering for 
$\mathrm{MC}$ is almost the same as in the vacuum but changes for the $\mathrm{MCH}^{+} \mathrm{Cl}^{-}$conformers. This behavior is observed in both theoretical schemes and for both basis sets. The stability of MC conformers as predicted in water is in agreement with earlier literature.[25] We have already demonstrated[25] that the planar forms of MC experience greater energetic reductions in water than the closed SP form implying that the chelation effect still dominates making the planar forms more stable than the twisted (dihydroindole and benzene ring are almost perpendicular) conformers. This observation is also made in case of $\mathrm{MCH}^{+} \mathrm{Cl}^{-}$where we see higher energetic changes. The stabilization of the ion-pair conformers $\mathrm{MCH}^{+} \mathrm{Cl}^{-}$is larger than the neutral $\mathrm{MC}$ molecules. We explain this by the fact that the twisted forms have a higher degree of polar zwitterionic character. Thus we expect that the stabilizing solute-solvent interactions favor an enlargement of the atomic net charges, especially in the protonated molecule. Overall, our results show that a PCM model is able to describe the conformer dependent differences between the calculations in vacuum and in solvent.

The dipole moments of the different MC conformers are computed using the two adopted basis sets; they are compared in Table 4. The results for MC show good agreement with the findings of earlier experimental[30] and theoretical investigations.[18] Employing a large basis set with polarization and diffuse functions leads to an increase in the dipole moment of almost all of the MC conformers. The required enlarged charge separation between the merocyanine atoms can be seen in the partial atomic charges of Table 5. The variation of dipole moments of the different moieties in water is also presented in Table 4. Relative to vacuum, a constant increase of about 6-8 D is observed for the stable MC conformers when the molecule is contained in water. It is useful to note that the conformers with a very high dipole moment have an energetically more favorable structure in water.

Let us present the partial charges on the atoms of the open form conformers using the different basis sets with DFT. Table 5 shows the results for two of the stable merocyanine moieties, TTC and TTT, both for the neutral and protonated forms, in vacuum and in water. The extended basis set $6-311 G(d, p)++$ shows certain differences in the charge distribution relative to the 6-31G(d) basis. Presence of polarization and diffuse functions enables an improved description of the valence electrons in quantum chemical calculations, as discussed above. With the larger basis set we observe electron distributions with enlarged charge separations in the conformer. The polarity 
of water induces stronger electrostatic effects through enhanced Coulomb interactions. The existence of a strongly zwitterionic character, which is described in some literature predicting integral positive and negative charges for $\mathrm{N} 2$ and $\mathrm{O} 8$ respectively, is neither evident for $\mathrm{MC}$ nor for $\mathrm{MCH}^{+} \mathrm{Cl}^{-}$.[31,32] The $\mathrm{N} 2$ atom has a small but a positive charge while the $\mathrm{O} 8$ atom has a negative partial charge.

Typical bond lengths in the TTC and TTT configurations of $\mathrm{MC}$ and $\mathrm{MCH}^{+} \mathrm{Cl}^{-}$in the gas phase and in water are reported in Table 6. The bond lengths correspond to the atom pairs labeled in Fig. 1. We concentrate on the differences in the results obtained with the two basis sets in DFT methods. Our vacuum calculations predict the characteristics of conjugated, i.e. non-alternating, $\mathrm{C}-\mathrm{C}$ bond lengths for the triple $\mathrm{C} 3-\mathrm{C} 4, \mathrm{C} 4-\mathrm{C} 5$ and $\mathrm{C} 5-\mathrm{C} 6$. In water the terminal bonds are elongated relative to the vacuum geometry. The opposite trend is found for the C4-C5 bond length, which is reduced in water. This effect is observed in $\mathrm{MC}$ but the behavior is completely different for the protonated case. The $\mathrm{C} 3-\mathrm{C} 4$ and $\mathrm{C} 5-\mathrm{C} 6$ bonds are weaker while $\mathrm{C} 4-\mathrm{C} 5$ is stronger in TTC conformer of $\mathrm{MCH}^{+} \mathrm{Cl}^{-}$. This is completely opposite to the effect observed in case of TTC conformer of MC in vacuum. However, in case of TTT conformer of $\mathrm{MCH}^{+} \mathrm{Cl}^{-}$in vacuum $\mathrm{C} 4-\mathrm{C} 5$ and $\mathrm{C} 5-\mathrm{C} 6$ bonds are relatively weaker than $\mathrm{C} 3-\mathrm{C} 4$. We also observe that the presence of neighboring water molecules in $\mathrm{MC}$ weakens the $\mathrm{C} 7-\mathrm{O} 8$ bond. Based on the increased stability of these structures in water, we conjecture that the molecular stability imparted by hydrogen bonding decreases the strength of the C7-O8 bond. Such a competition between intermolecular hydrogen bonds and intramolecular "covalent" bonds in water has been discussed in a recent path integral study.[33]

\section{Conclusions}

The relative stability of the six conformers of merocyanine and its protonated forms has been investigated using ab-initio MP2 and DFT calculations. Our results show that the choice of the basis set level seems to be not a major factor when calculating the structural properties and the electronic energies. The inclusion of polarization and diffuse functions in the basis set provides nevertheless an improved distribution of charges, bond lengths and the energetic ordering. The differences in the energetic ordering of the conformers obtained from the different computational approaches cannot be overlooked, and future simulations beyond HF MP2 might offer more accurate results, especially when removing the basis set superposition error in the protonated 
systems. $\mathrm{MC}$ and $\mathrm{MCH}^{+} \mathrm{Cl}^{-}$conformers in solution are strongly stabilized and their dipole moments are significantly enhanced, which we attribute to the solute-solvent interactions. In general, the structures that have highest dipole moments show the strongest reduction in their energies. Both the neutral and protonated forms do not exhibit any zwitterionic character in their electronic structures.

\section{Acknowledgements}

RS and GB thank the Center for Nanoscale Materials (supported by the U. S. Department of Energy, Office of Science, Office of Basic Energy Sciences, under Contract No. DE-AC0206CH11357) at the Argonne National Laboratory for use of the high-performance computing cluster Carbon. We are grateful to Prof. Dr. M. Biesalski and Dr. H. Schenderlein (Ernst-Berl Institut für Technische und Makromolekulare Chemie, Technische Universität Darmstadt) for fruitful discussions in the early phase of our work. RS would like to thank the College of Engineering for the Dean's Fellowship. 


\section{References}

[1] V.A. Krongauz, E.S. Goldburt, Macromolecules 14 (1981) 1382.

[2] E. Goldburt, F. Shvartsman, S. Fishman, V. Krongauz, Macromolecules 17 (1984) 1225.

[3] W. Tian, J. Tian, Dyes and Pigments 105 (2014) 66.

[4] T. Yoshida, A. Morinaka, N. Funakoshi, Thin Solid Films 162 (1988) 343.

[5] I. Willner, Bioorganic Photochemistry Vol2:Biological Applications of Photochemical Switches, John Wiley and Sons, Newyork.

[6] G. Berkovic, V. Krongauz, V. Weiss, Chemical Reviews 100 (2000) 1741.

[7] N. Lu, V. Nguyen, S. Kumar, A. McCurdy, Journal of Organic Chemistry 70 (2005) 9067.

[8] F. Raymo, M. Tomasulo, Chemistry European Journal 12 (2006) 3186.

[9] B.L. Feringa, Molecular Switches, Wiley-VCH, Newyork, 2003.

[10] D. Gust, T. Moore, A. Moore, Chemical Communications 11 (2006) 1169.

[11] N. Tamai, H. Miyasaka, Chemical Reviews 100 (2000) 1875.

[12] V. Malatesta, C. Neri, M.L. Wis, L. Montanari, R. Millini, Journal of the American Chemical Society 119 (1997) 3451.

[13] Y. Sheng, J. Leszczynski, A.a. Garcia, R. Rosario, D. Gust, J. Springer, The Journal of Physical Chemistry B 108 (2004) 16233.

[14] F. Liu, K. Morokuma, Journal of the American Chemical Society 135 (2013) 10693.

[15] M. Inouye, M. Ueno, T. Kitao, K. Tsuchiya, Journal of the American Chemical Society 112 (1990) 8977.

[16] J. Whelan, D. Abdallah, K. Piskorz, J.T. Wojtyk, J.M. Dust, J.M. Nunzi, S. Hoz, E. Buncel, Physical Chemistry Chemical Physics 14 (2012) 13684.

[17] M. Hammarson, J.R. Nilsson, S. Li, T. Beke-Somfai, J. Andreasson, Journal of Physical Chemistry. B 117 (2013) 13561.

[18] R. Ganesan, F. Remacle, Theoretical Chemistry Accounts 131 (2012) 1255.

[19] L.d. Leon, M.C. Biewer, Tetrahedron Letters 41 (2000) 3527.

[20] C. Wohl, D. Kuciauskas, Journal of Physical Chemistry B 109 (2005) 22186.

[21] L. Poisson, K. Raffael, B. Soep, J. Mestdagh, G. Buntinx, Journal of the American Chemical Society 128 (2006) 3169.

[22] S. Stitzel, R. Byrne, D. Diamond, Journal of Materials Science 41 (2006) 5841. 
[23] T. Tamaki, M. Sakuragi, K. Ichimura, K. Aoki, Chemical Physics Letters 161 (1989) 23.

[24] F. Maurel, J. Aubard, P. Millie, J.P. Dognon, M. Rajzmann, R. Guglielmetti, A. Samat, Journal of Physical Chemistry A 110 (2006) 4759.

[25] G. Balasubramanian, J. Schulte, F. Müller-Plathe, M.C. Böhm, Chemical Physics Letters 554 (2012) 60.

[26] A.D. Becke, The Journal of Chemical Physics 98 (1993) 5648.

[27] C. Møller, M.S. Plesset, Physical Review 46 (1934) 618.

[28] S. Miertus̃, J. Tomasi, Chemical Physics 65 (1982) 239.

[29] U.C. Singh, P.A. Kollman, Journal of Computational Chemistry 5 (1984) 129.

[30] M. Levitus, G. Glasser, D. Neher, P. Aramendía, Chemical Physics Letters 277 (1997) 118.

[31] Z. Sainudeen, P.C. Ray, The Journal of Physical Chemistry A 109 (2005) 9095.

[32] C. Salemi-Delvaux, B. Luccioni-Houze, G. Baillet, G. Giusti, R. Guglielmetti, Journal of Photochemistry and Photobiology A: Chemistry 91 (1995) 223.

[33] R. Ramírez, J.K. Singh, F. Müller-Plathe, M.C. Böhm, The Journal of Chemical Physics 141 (2014) 204701. 


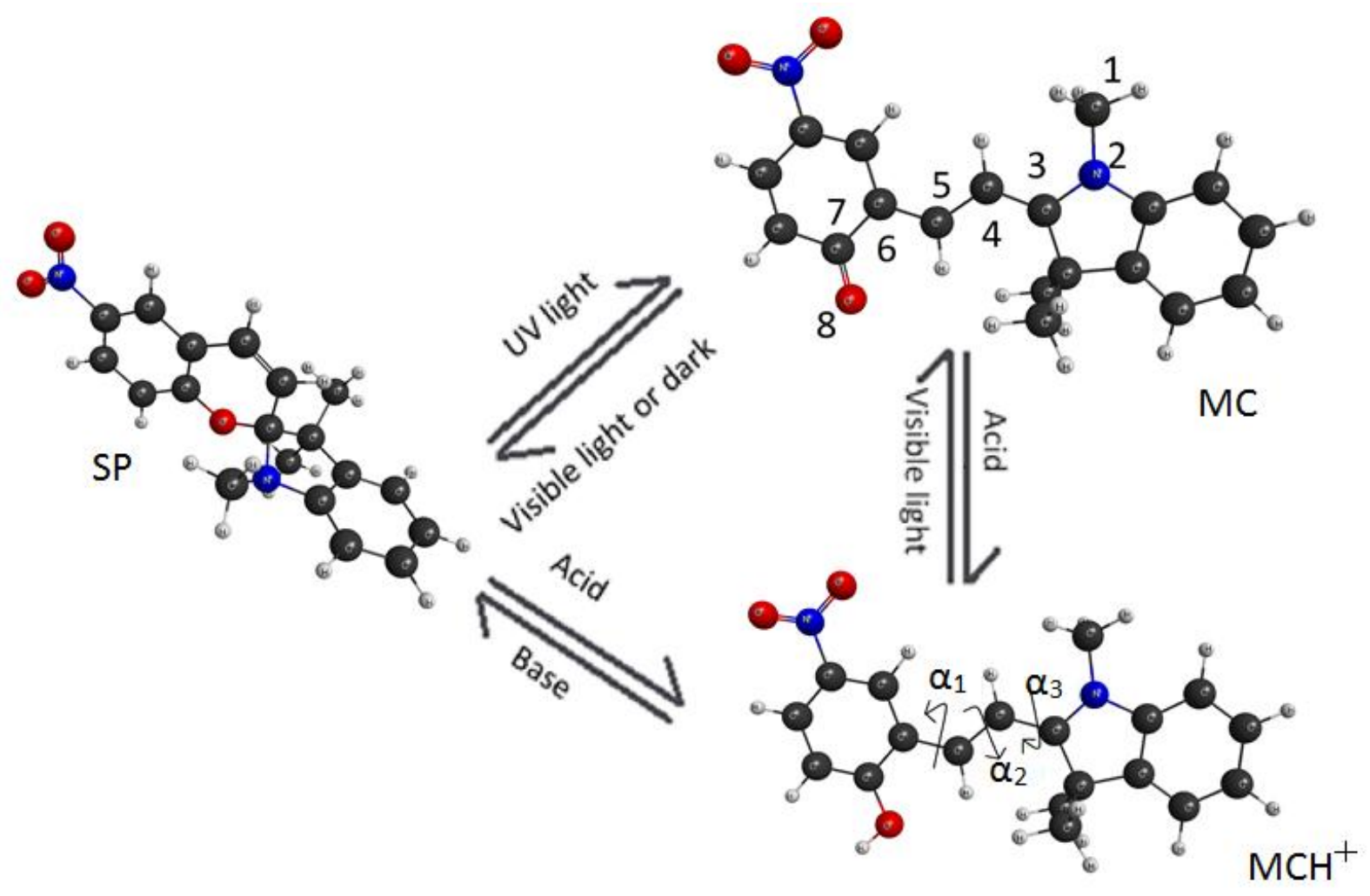

Figure 1. Reversible photoisomerization process showing that spiropyran derivatives can exist in three states: SP, MC and protonated $\mathrm{MCH}^{+}$. 
Table 1. Ground state energies $(\mathrm{kJ} / \mathrm{mol})$ of the different MC conformers relative to the most stable MC conformer, TTC.

\begin{tabular}{ccccccccc}
\hline \multicolumn{7}{c}{ MC } \\
\hline & $\begin{array}{c}\text { 6-31G(d) } \\
\text { Vacuum }\end{array}$ & $\begin{array}{c}\text { 6-311G(d,p)++ } \\
\text { Vacuum }\end{array}$ & $\begin{array}{c}\text { 6-31G(d) - } \\
\text { Water }\end{array}$ & $\begin{array}{c}\text { 6-311G(d,p)++ } \\
\text { - Water }\end{array}$ \\
\cline { 2 - 10 } & $\begin{array}{c}\text { DFT } \\
{[25]}\end{array}$ & MP2 & $\begin{array}{c}\text { DFT } \\
{[\mathbf{2 5}]}\end{array}$ & MP2 & DFT [25] & MP2 & DFT & MP2 \\
\hline TTC & 0 & 0 & 0 & 0 & 0 & 0 & 0 & 0 \\
CCC & 27.9 & 1.9 & 26.7 & -9.4 & 31.8 & 4.6 & 32.7 & 5.6 \\
TTT & 6.7 & 8.6 & 6.0 & 4.2 & 3.8 & 6.3 & 2.5 & 6.7 \\
CTC & 8.7 & 8.7 & 8.7 & 8.4 & 5.9 & 6.4 & 1.9 & 7.2 \\
CTT & 0.03 & 14.7 & 0.2 & 15.0 & -0.1 & 12.9 & 8.0 & 11.7 \\
TCC & 27.8 & 67.7 & 27.5 & 59.2 & 32.3 & 62.3 & 75.1 & 58.9 \\
\hline
\end{tabular}


Table 2. Ground state energies $(\mathrm{kJ} / \mathrm{mol})$ of the different conformers of $\mathrm{MCH}^{+} \mathrm{Cl}^{-}$and $\mathrm{MCH}^{+}$ relative to the TTC conformer in the corresponding series.

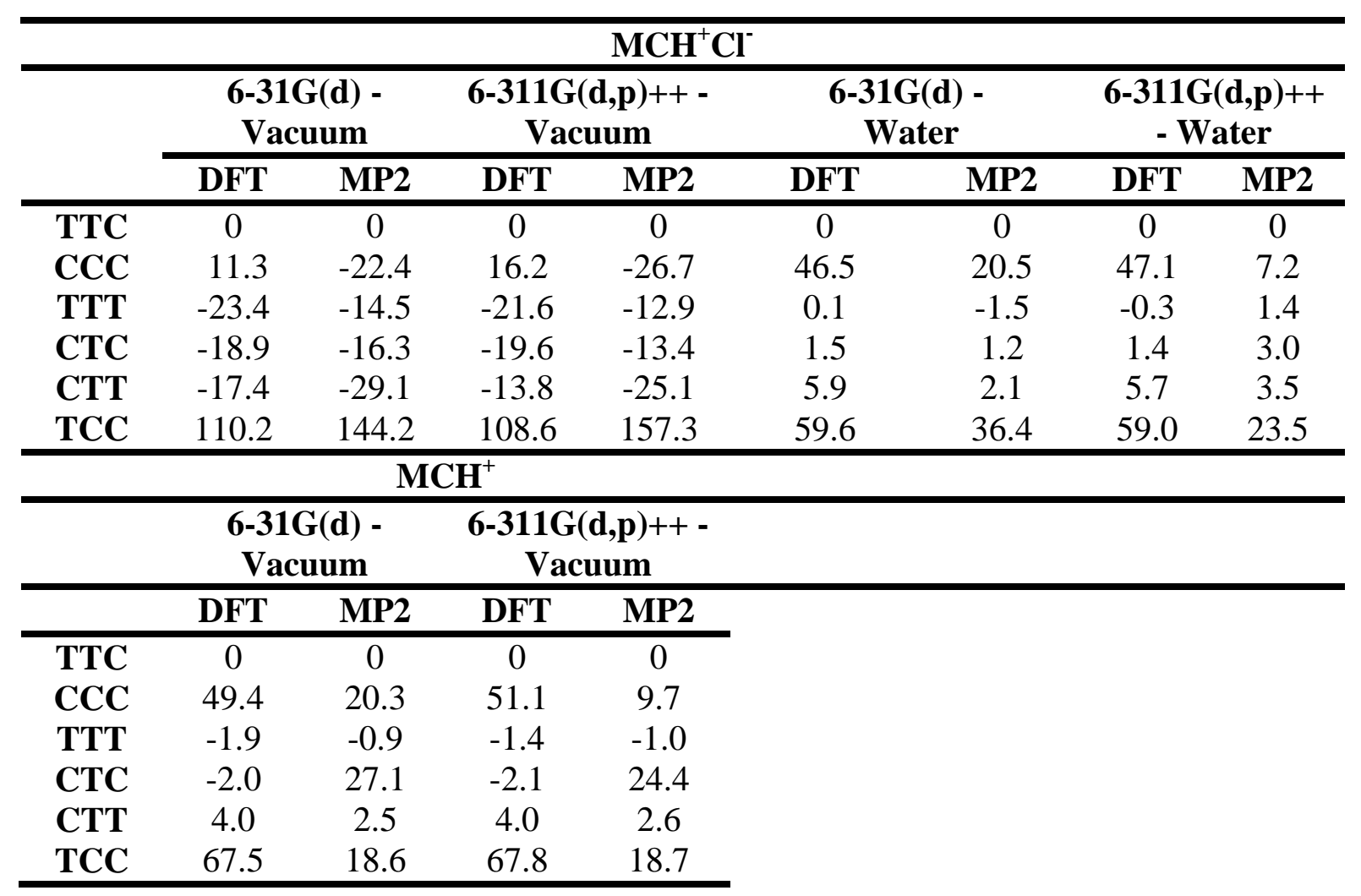


Table 3. Stabilization energies $(\mathrm{kJ} / \mathrm{mol})$ of the different conformers of $\mathrm{MC}$ and $\mathrm{MCH}^{+} \mathrm{Cl}^{-}$in water relative to the corresponding energies in vacuum.

\begin{tabular}{|c|c|c|c|c|c|c|c|c|}
\hline \multicolumn{5}{|c|}{$\overline{M C}$} & \multicolumn{4}{|c|}{$\mathrm{MCH}^{+} \mathrm{Cl}^{-}$} \\
\hline & \multicolumn{2}{|c|}{$\begin{array}{l}\text { 6-31G(d) - } \\
\text { Water }\end{array}$} & \multicolumn{2}{|c|}{$\begin{array}{c}\text { 6-311G(d,p)++ - } \\
\text { Water }\end{array}$} & \multicolumn{2}{|c|}{$\begin{array}{l}\text { 6-31G(d) - } \\
\text { Water }\end{array}$} & \multicolumn{2}{|c|}{$\begin{array}{c}\text { 6-311G(d,p)++ } \\
\text { - Water }\end{array}$} \\
\hline & $\begin{array}{l}\text { DFT[2 } \\
5]\end{array}$ & MP2 & $\begin{array}{l}\text { DFT }[2 \\
5]\end{array}$ & MP2 & DFT & MP2 & DFT & MP2 \\
\hline TTC & -60.0 & -55.0 & -72.3 & -62.4 & -116.9 & -64.8 & -136.5 & -33.5 \\
\hline $\mathrm{CCC}$ & -56.2 & -52.3 & -66.7 & -56.7 & -81.8 & -22.0 & -105.6 & 0.4 \\
\hline TTT & -63.0 & -57.2 & -75.6 & -65.9 & -93.4 & -52.0 & -115.3 & -19.2 \\
\hline CTC & -62.7 & -57.2 & -75.1 & -65.6 & -96.5 & -47.4 & -115.6 & -17.2 \\
\hline CTT & -60.2 & -56.7 & -72.6 & -65.0 & -93.6 & -33.7 & -117.0 & -4.9 \\
\hline TCC & -55.5 & -159.2 & -66.6 & -176.3 & -167.5 & -172.6 & -186.1 & -167.4 \\
\hline
\end{tabular}


Table 4. Dipole moment (in Debye) of the different MC conformers as obtained from DFT [25] and MP2 calculations using different basis sets.

\begin{tabular}{ccccccc}
\hline & \multicolumn{2}{c}{ 6-31G(d) - Vacuum } & \multicolumn{2}{c}{ 6-311G(d,p)++ - Vacuum } & \multicolumn{2}{c}{ 6-31G(d) - Water } \\
\cline { 2 - 7 } & DFT [25] & MP2 & DFT [25] & MP2 & DFT [25] & MP2 \\
\hline TTC & 11.82 & 10.95 & 12.48 & 11.22 & 18.97 & 19.65 \\
CCC & 10.97 & 10.36 & 11.58 & 10.42 & 16.83 & 16.33 \\
TTT & 10.89 & 9.53 & 11.44 & 8.49 & 18.11 & 18.86 \\
CTC & 11.50 & 9.52 & 12.16 & 10.29 & 18.58 & 18.66 \\
CTT & 11.82 & 9.60 & 11.60 & 9.79 & 18.97 & 19.04 \\
TCC & 10.91 & 9.60 & 11.54 & 9.98 & 16.76 & 6.44 \\
\hline
\end{tabular}


Table 5. The partial charges $(e)$ in $\mathrm{MC}$ and $\mathrm{MCH}^{+} \mathrm{Cl}^{-}$on the atoms in the vicinity of the original spiro center (C3) of merocyanine in the neutral and protonated forms, as labeled in Fig. 1. The data refer to DFT calculations with different basis sets in vacuum and in water.

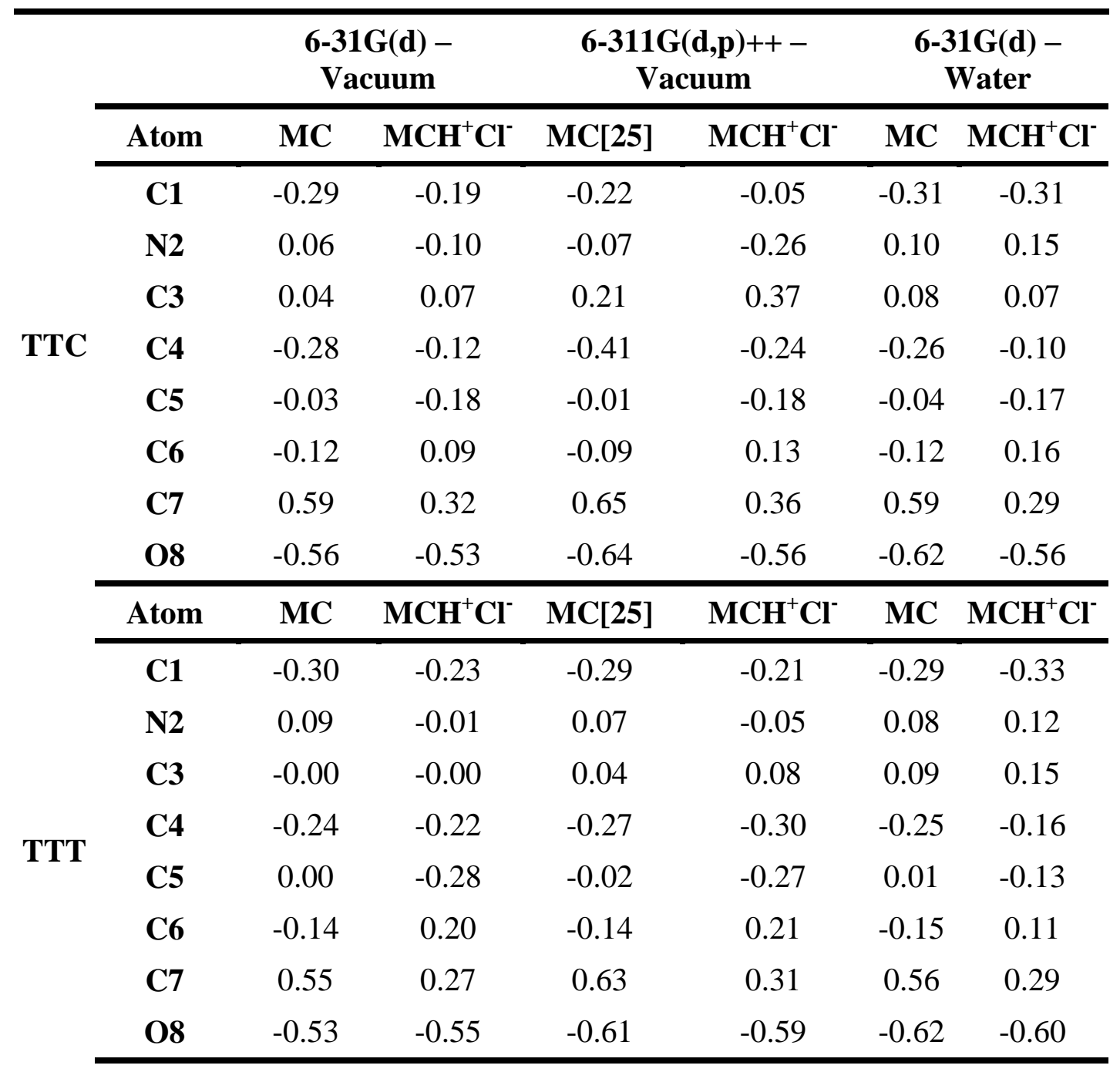


Table 6. The bond lengths (in pm) between the atom pairs, as labeled in Fig. 1, around the original spiro-carbon atom (C3) of merocyanine in the neutral and protonated forms. The data refer to DFT calculations with different basis sets in vacuum and in water.

\begin{tabular}{|c|c|c|c|c|c|c|c|}
\hline & & \multicolumn{2}{|c|}{$\begin{array}{l}\text { 6-31G(d) - } \\
\text { Vacuum }\end{array}$} & \multicolumn{2}{|c|}{$\begin{array}{c}\text { 6-311G }(d, p)++- \\
\text { Vacuum }\end{array}$} & \multicolumn{2}{|c|}{ 6-31G(d) - Water } \\
\hline & Bond & MC & $\mathrm{MCH}^{+} \mathrm{Cl}^{-}$ & MC[25] & $\mathrm{MCH}^{+} \mathrm{Cl}^{-}$ & MC & $\mathrm{MCH}^{+} \mathrm{Cl}^{-}$ \\
\hline & C1-N2 & 145.7 & 145.4 & 145.9 & 145.6 & 146.4 & 147.1 \\
\hline & N2-C3 & 136.6 & 139.5 & 136.1 & 136.5 & 134.8 & 132.6 \\
\hline \multirow{9}{*}{ TTC } & C3-C4 & 138.8 & 148.0 & 138.9 & 146.1 & 140.7 & 143.4 \\
\hline & C4-C5 & 140.2 & 134.7 & 139.7 & 134.7 & 138.5 & 136.1 \\
\hline & C5-C6 & 140.3 & 146.6 & 140.2 & 146.2 & 142.3 & 145.5 \\
\hline & C6-C7 & 149.1 & 142.0 & 148.8 & 149.1 & 148.1 & 141.7 \\
\hline & C7-08 & 124.4 & 136.0 & 124.1 & 135.9 & 125.3 & 135.9 \\
\hline & Bond & MC & $\mathrm{MCH}^{+} \mathrm{Cl}^{-}$ & $\mathrm{MC}[25]$ & $\mathrm{MCH}^{+} \mathrm{Cl}^{-}$ & MC & $\mathrm{MCH}^{+} \mathrm{Cl}^{-}$ \\
\hline & C1-N2 & 145.5 & 144.6 & 145.6 & 146.8 & 148.3 & 146.9 \\
\hline & N2-C3 & 136.8 & 139.1 & 136.3 & 145.1 & 134.8 & 132.4 \\
\hline & C3-C4 & 138.7 & 135.8 & 138.7 & 135.8 & 140.6 & 143.5 \\
\hline \multirow[t]{4}{*}{ TTT } & C4-C5 & 140.3 & 147.3 & 139.9 & 147.3 & 138.4 & 135.8 \\
\hline & C5-C6 & 139.5 & 150.8 & 139.5 & 150.9 & 141.8 & 145.5 \\
\hline & C6-C7 & 149.5 & 141.2 & 149.3 & 140.6 & 148.4 & 141.5 \\
\hline & C7-08 & 123.9 & 136.1 & 123.6 & 136.4 & 124.9 & 136.0 \\
\hline
\end{tabular}


Table of Contents Image

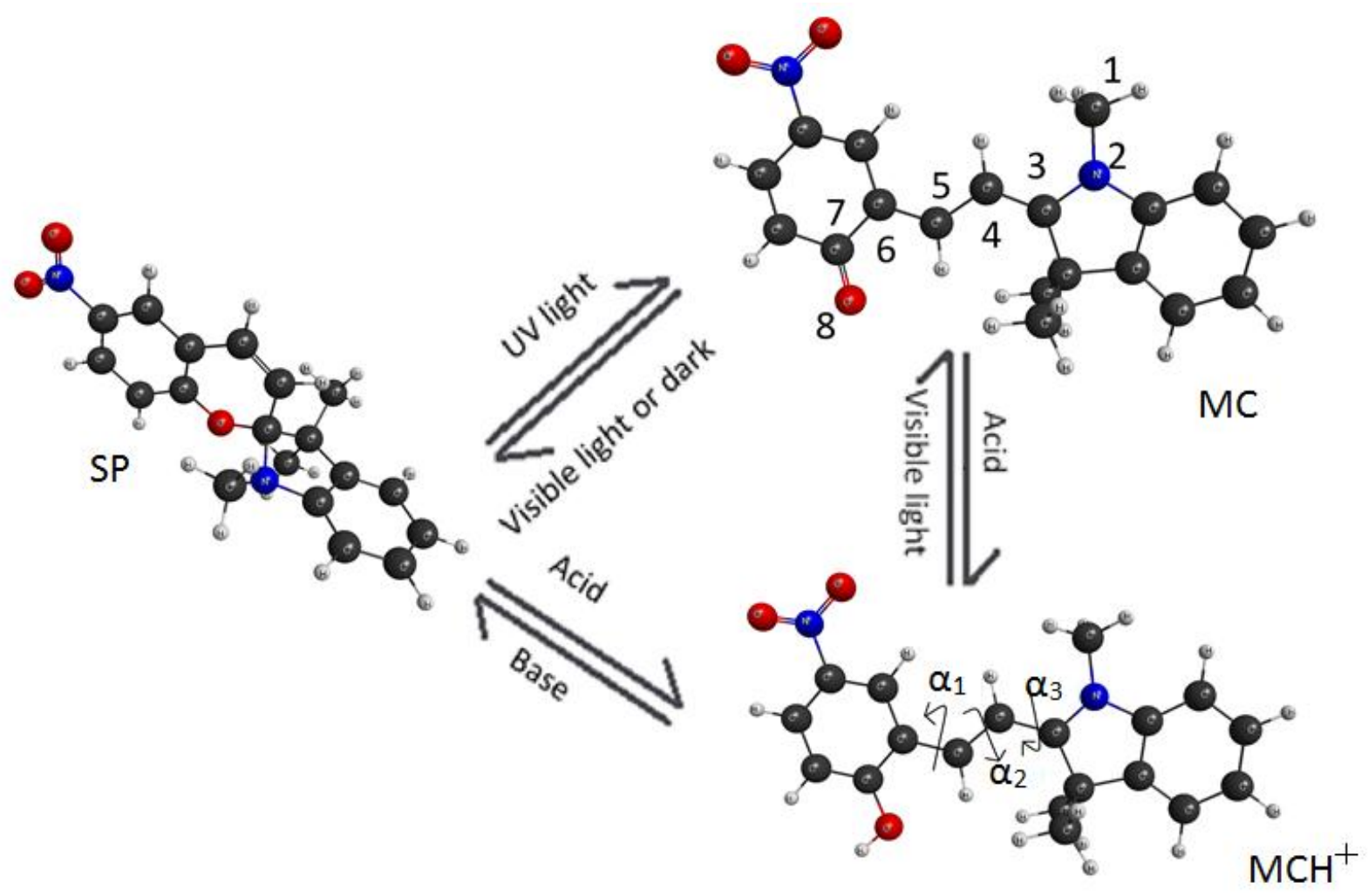

